\title{
Ocorrência de enteroparasitas em alface crespa (Lactuca sativa) de cultivo convencional comercializadas em supermercados e hortas comunitárias de Teresina, Piauí
}

\author{
Occurrence of enteroparasites in curly lettuce (Lactuca sativa) of conventional cultivation \\ sold in supermarkets and community gardens of Teresina, Piauí
}

Ocurrencia de enteroparásitos en lechuga rizada (Lactuca sativa) de cultivo convencional que se vende en supermercados y jardines comunitarios de Teresina, Piauí

Jurecir da Silva ${ }^{1,2 *}$, Vanessa Gomes de Moura ${ }^{1}$, Manoel de Jesus Marques da Silva1 ${ }^{1}$, Camila de Carvalho Chaves ${ }^{1}$, Adayane Vieira Silva1, Poliana Brito de Sousa1, Marcelo Cardoso da Silva Ventura ${ }^{1}$, Jossuely Rocha Mendes ${ }^{1}$, Rômulo Oliveira Barros ${ }^{1}$, Elaine Ferreira do Nascimento², Jessica Pereira dos Santos ${ }^{2}$, Simone Mousinho Freire ${ }^{3}$.

\section{RESUMO}

Objetivo: Este estudo versa sobre a qualidade higiênica da alface comercializada na cidade de Teresina, Piauí, por horticultores e supermercados. Métodos: No período de janeiro a abril de 2016, foram coletadas aleatoriamente amostras de alfaces em hortas comunitárias e supermercados da cidade e conduzidas ao Laboratório de Parasitologia do Instituto Federal do Piauí para análise parasitológica seguindo a metodologia de Hoffman, Pons e Janer e Ritchie modificado. A positividade das amostras foi confirmada pela visualização de formas evolutivas de enteroparasitas em um dos testes. Resultados: $O$ índice de contaminação por formas parasitárias foi de $66,5 \%$ (21/32) nas amostras analisadas. Sendo $64,7 \%(11 / 17)$ e $66,5 \%$ (10/15) para os supermercados e hortas comunitárias respectivamente. Os protozoários encontrados foram os comensais Endolimax nana, Entamoeba coli, lodamoeba butschlii e os patogênicos, Entamoeba histolytica/díspar, Giardia sp. e Eimeria sp. Dentre os helmintos, Ascaris sp, Ancylostoma $s p$ e Strongyloides $s p$ foram ocorrentes. A presença de enteroparasitas nas alfaces aponta que as condições higiênico-sanitárias são insatisfatórias ao longo da cadeia produtiva aumentando o risco de infecção pelos consumidores. Conclusão: As alfaces comercializadas em Teresina-PI estão contaminadas, tais dados sugerem uma melhor estratégia de higienização para o consumo de alface pela população da área de estudo.

Palavras-chave: Alface, Doenças Parasitárias, Saúde Pública.

\begin{abstract}
Objective: This study deals on the hygienic quality of lettuce commercialized in the city of Teresina, Piauí, by small horticulturists and supermarkets. Methods: In the period of January to April 2016aleatory samples of lettuce were collected in community horticulture yards and supermarkets and, then, taken to the Laboratory of Parasitology of Federal Institute of Piauí for parasitological analysis, following the modified methodology of Hoffman, Pons, and Janer. The positivity of the samples was confirmed by visualization of evolutive forms of enteroparasites in one of the tests. Results: The rate of contamination by parasitary forms was $66.5 \%(21 / 32)$ of the samples analyzed, being $64.7 \%(11 / 17)$ and $66.5 \%$ (10/15) for supermarkets and community horticulture yards respectively. The protozoa found were the commensals Endolimax nana, Entamoeba coli, lodamoeba butschlii, and the pathogenic Entamoeba histolytica/díspar, Giardia sp., and Eimeria sp. Among the helminths, Ascaris sp, Ancylostoma sp, and Strongyloides sp occurred. The presence of enteroparasites in lettuce points that hygienic-sanitary conditions along the productive chain is not satisfactory, increasing the risk of infection by the consumers. Conclusion: Lettuces commercialized in Teresina-PI are contaminated. Such data suggest that a better hygienization strategy should be taken for the consumption of lettuce by the population of the studied area.
\end{abstract}

Keywords: Lettuce, Parasitic Diseases, Public Health.

1'Instituto Federal do Piauí (IFPI), Escritório Regional Fiocruz Piauí, Teresina-PI. *E-mail: jurecir.silva@ifpi.edu.br

${ }^{2}$ Escritório Regional Fiocruz Piauí, Teresina- PI

${ }^{3}$ Universidade Estadual do Piauí (UESPI), Teresina-PI. 


\section{RESUMEN}

Objetivo: Este estudio aborda la calidad higiénica de la lechuga comercializada en la ciudad de Teresina, Piauí, por jardineros y supermercados. Métodos: de enero a abril de 2016, se recolectaron muestras de lechuga al azar de los jardines y supermercados comunitarios de la ciudad y se llevaron al Laboratorio de Parasitología del Instituto Federal de Piauí para un análisis parasitológico siguiendo la metodología de Hoffman, Pons y Janer y Ritchie modificado. . La positividad de las muestras se confirmó mediante la visualización de formas evolutivas de enteroparásitos en una de las pruebas. Resultados: La tasa de contaminación por formas parasitarias fue de $66.5 \%$ (21/32) en las muestras analizadas. De los cuales $64.7 \%$ $(17 / 11)$ y $66.5 \%(15 / 10)$ para supermercados y jardines comunitarios respectivamente. Los protozoos encontrados fueron los comensales Endolimax nana, Entamoeba coli, lodamoeba butschlii y los patógenos, Entamoeba histolytica / dispar, Giardia sp. y Eimeria sp. Entre los helmintos, se encontraron Ascaris sp, Ancylostoma sp y Strongyloides sp. La presencia de enteroparásitos en la lechuga indica que las condiciones higiénico-sanitarias son insatisfactorias a lo largo de la cadena de producción, lo que aumenta el riesgo de infección por parte de los consumidores. Conclusión: las lechugas comercializadas en Teresina-PI están contaminadas, tales datos sugieren una mejor estrategia de higiene para el consumo de lechuga por parte de la población del área de estudio.

Palabras llaves: Lechuga, Enfermedad parasitaria, Salud pública.

\section{INTRODUÇÃO}

Por serem ricas em sais minerais, vitaminas, fibras alimentares e baixa caloria, as hortaliças consumidas in natura são amplamente recomendadas como parte de uma alimentação saudável dentre elas a mais comercializada e consumida no Brasil é a alface (Lactuca sativa), uma hortaliça folhosa pertencente à família Asteraceae, assim como a escarola, a alcachofra, a chicória e o almeirão (VIEIRA JN et al. 2013; SOARES B e CANTOS GA, 2005). É considerada importante fonte de sais minerais, tais como: enxofre, ferro, cálcio, fósforo e de vitaminas ( $\mathrm{A}$ e $\mathrm{C}$ ), também possui a função de desintoxicar e alcalinizar, além do efeito diurético, calmante e laxante (GONDIM A, 2010).

Atualmente, a busca por alimentos saudáveis, principalmente os vegetais, tem sido significativa em todo o mundo, decorrente da conscientização da população em adquirir bons hábitos alimentares. Devido à sua facilidade de produção e acesso, a alface é amplamente consumida na forma in natura em saladas, sanduiches e muito utilizada nas decorações de pratos (TAKAYANAGUI OM et al., 2006; SANTANA LRR et al., 2006). Segundo Freitas AA et al., (2004) e Vieira JN et al., (2013), na busca por uma alimentação saudável que é encontrada principalmente em alface e outros vegetais, os consumidores se expõem aos riscos de contraírem infecções por enteroparasitas, pois, quando consumidas cruas sem higienização adequada podem conter ovos e larvas de helmintos, bem como, cistos e oocistos de protozoários servindo de via de transmissão.

Para manter suas propriedades nutricionais a alface não passa por nenhum tratamento térmico, tornandoa um excelente carreador de enteroparasitas se estiver contaminada. Essas contaminações podem ocorrer de diversas formas, como a utilização de água contaminada com fezes de origem animal e humana ou solo contaminado devido ao uso de adubo orgânico com dejetos fecais (SANTANA LRR et al., 2006).

A contaminação também pode ocorrer em etapas da cadeia produtiva; na coleta, no transporte, no armazenamento e na comercialização. Um fator importante na veiculação de enteroparasitas é a falta de higiene pessoal no momento da manipulação dos alimentos. Indivíduos infectados e assintomáticos que manipulam alimentos são na maioria das vezes uma excelente fonte de contaminação e disseminação dessas infecções (COSTANTIN BS et al., 2013; ADANI AAV e DUTRA MBL,2011; COELHO WLD et al., 2009).

As enteroparasitoses apesar de antigas perpetuam-se com um sério problema de saúde pública no Brasil. O clima tropical, baixa condição socioeconômica, más condições de saneamento e acesso a saúde favorecem à incidência e prevalência de doenças parasitárias tanto em áreas rurais quanto nas urbanas. Essas infecções são deletérias à saúde humana, manifestando consequências como a diarreia, anemia, hemorragia, desequilíbrios nutricionais e em condições mais graves, casos de óbito (NEVES DP, 2011). 
Diversos estudos recentes e antigos demonstram em seus resultados um índice elevado de contaminação em alfaces por parasitas entéricos, como protozoários e helmintos. As largas folhas flexíveis, justapostas e estrutura compacta apresentam maior possibilidade de contaminação, pois, durante o seu cultivo ocorre um contato maior com o solo poluído e a água, consequentemente uma maior adesão das formas evolutivas parasitárias, conferindo uma maior resistência aos métodos de higienização (MESQUITA VCL et al., 1999; FALAVIGNA LM et al., 2005; SANTOS AS e MERLINI LS, 2010; MELO ACFL et al., 2011; SANTARÉM VA et al.,2012; MESQUITA DR et al., 2015).

Dentre as principais parasitoses intestinais veiculadas por vegetais consumidos in natura, podem-se citar as helmintíases: ascaridíase, teníase, ancilostomíase e estrongiloidíase, e as protozooses: giardíase e amebíase (GREGÓRIO DS et al., 2012). Diante do exposto, a análise laboratorial da sanidade parasitológica das hortaliças torna-se uma ferramenta importante no controle de transmissão das enteroparasitoses para o setor da saúde pública, uma vez que pode fornecer dados sobre as condições higiênicas-sanitárias envolvidas nas etapas da cadeia produtiva desses produtos, demonstrando os riscos de infecção aos quais os consumidores se expõem e propiciar medidas profiláticas adequadas para a descontaminação desses alimentos (FERRO JJV et al., 2012).

Objetivou-se com este estudo avaliar a possível presença de diferentes formas evolutivas de parasitos de importância médica em amostras de alfaces (Lactuca sativa) da classe crespa, comercializadas em hortas comunitárias e supermercados da cidade de Teresina, Piauí.

\section{MÉTODOS}

Trata-se de uma pesquisa de campo realizada no período de janeiro a abril de 2016, onde houve análise de amostras de alfaces cultivadas convencionalmente em três hortas comunitárias e quatro supermercados segundo a metodologia de Hoffman, Pons e Janer e Ritchie com adaptações (ROCHA MO e MELO RT, 2011). As coletas das amostras de alfaces foram realizadas em duas hortas comunitárias na região sul e uma na região norte de Teresina - Piauí e quatro supermercados, sendo dois em cada região descrita. A escolha da região foi aleatória.

Foram definidos como unidades de análise as hortas e supermercados que oferecessem hortaliças de plantios convencionais. Para cada região foram identificados os supermercados que atendessem a esse critério sendo denominados de S1, S2, S3 e S4. O mesmo procedimento amostral foi adotado para as hortas comunitárias de cada bairro, onde foram coletadas amostras convencionais sendo denominadas de $\mathrm{H} 1, \mathrm{H} 2 \mathrm{e}$ H3.A unidade de amostra foi o "pé" de alface (Lactuca sativa), do tipo "crespa", independentemente de tamanho e peso, cultivada pelo método convencional. As amostras foram selecionadas aleatoriamente, conforme a disponibilidade para a comercialização. A seleção das amostras foi organizada conforme Tabela 1.

Tabela 1- Locais de coleta e número de alfaces coletadas em Supermercados e Hortas comunitárias de Teresina-PI.

\begin{tabular}{ccc}
\hline Grupos & Locais & № Amostras \\
\hline & $\mathrm{S} 1$ & 05 \\
Grupo I (Supermercados) & $\mathrm{S} 2$ & 04 \\
& $\mathrm{~S} 3$ & 04 \\
& $\mathrm{~S} 4$ & 04 \\
& Total & $\mathbf{1 7}$ \\
Grupo II (Hortas) & $\mathrm{H} 1$ & 05 \\
& $\mathrm{H} 2$ & 05 \\
Total Geral de Amostras & $\mathrm{H} 3$ & 05 \\
\hline
\end{tabular}

Fonte: Silva J, Moura VG, Silva MJM, et al., 2016. 
As amostras foram coletadas pela manhã entre $7 \mathrm{~h}$ e $9 \mathrm{~h}$ distribuídas em dias alternados na semana. Nos supermercados as amostras (pés de alface) foram recolhidas e manuseadas com luvas de procedimento e selecionadas na prateleira de forma aleatória. As amostras foram acondicionadas de forma individual em sacos de polietileno e cada saco foi identificado e lacrado para o transporte. Nas hortas comunitárias, o próprio produtor acondicionava as amostras individualmente em sacos de polietileno, devidamente identificados e lacrados. Após a coleta, as amostras foram imediatamente encaminhadas para análise no Laboratório de Parasitologia do Instituto Federal do Piauí.

As amostras foram individualmente preparadas para análise no laboratório, utilizando-se luvas de procedimento e descartando-se as partes das alfaces impróprias para consumo (folhas deterioradas, queimadas e o talo). Em cubas de plástico tamanho $38 \mathrm{~cm} \times 25 \mathrm{~cm}$, cada amostra foi desfolhada e colocada num saco de polietileno de primeiro uso, e submetidas à lavagem e homogeneização com $350 \mathrm{ml}$ de água destilada por 5 minutos. Logo após, as folhas foram suspensas para drenagem do líquido e em seguida descartadas. A cada amostra as luvas de procedimento eram trocadas. A solução obtida da lavagem de cada amostra foi filtrada em gaze dobrada em quatro para o cálice de sedimentação espontânea e deixada em repouso para ser analisada entre 2 a 24 horas segundo a metodologia de Hoffman, Pons e Janer (ROCHA MO e MELO RT, 2011).

No dia seguinte, a análise do sedimento espontâneo foi realizada entre 19 e 20 horas, logo após foi deixado em repouso por mais duas horas, o líquido sobrenadante foi desprezado, transferindo-se cerca de $10 \mathrm{ml}$ do sedimento final para um tubo cônico de $15 \mathrm{ml}$. O tubo foi centrifugado durante três minutos a $1.500 \mathrm{rpm}$, o sobrenadante desprezado e ressuspenso o sedimento delicadamente baseando-se no método de Ritchie modificado (ROCHA MO e MELO RT, 2011). Foram pipetados $100 \mu \mathrm{l}$ do sedimento numa lâmina, corada com lugol e coberta com lamínula e analisada por meio de microscopia óptica nas objetivas de 10x e 40x. A leitura foi realizada por varredura segundo o método utilizado por Takayanagui OM et al., (2006). A positividade do teste foi confirmada pela visualização de formas evolutivas de parasitos (cistos e oocistos de protozoários e/ou larvas e ovos de helmintos). Os resultados obtidos foram submetidos a tratamento estatístico de análise na plataforma Microsoft $\Theta$ Exce/® 2016.

\section{RESULTADOS E DISCUSSÃO}

As amostras das hortas foram as mais comprometidas pelos agentes parasitários, contabilizando um total de $66,6 \%$ (10/15) de contaminação, ao passo que as amostras de alface coletadas dos supermercados tiveram presença de parasitos em 64,7\% (11/17), conforme demonstra a Tabela 2.

Tabela 2 - Positividade de enteroparasitos em amostras de alface coletadas em supermercados e hortas comunitárias em Teresina - PI.

\begin{tabular}{lccc}
\hline \multirow{2}{*}{ Supermercados } & \multicolumn{3}{c}{ Positividade das amostras } \\
\cline { 2 - 4 } S1 & AA & AP & $\%$ \\
S2 & 5 & 4 & $80 \%$ \\
S3 & 4 & 2 & $50 \%$ \\
S4 & 4 & 3 & $75 \%$ \\
TOTAL & 4 & 2 & $50 \%$ \\
Hortas Comunitárias & 17 & 11 & $64,7 \%$ \\
H1 & & & $60 \%$ \\
H2 & 5 & 3 & $80 \%$ \\
H3 & 5 & 4 & $60 \%$ \\
\hline TOTAL & 5 & 3 & $66,6 \%$ \\
\hline
\end{tabular}

Legenda: $\mathrm{AA}=$ Amostra Analisada; $\mathrm{AP}=$ Amostra Positiva.

Fonte: Silva J, Moura VG, Silva MJM, et al., 2016. 
Os resultados apresentados neste trabalho mostram que das 32 amostras estudadas, $66,5 \%(21 / 32)$ apresentaram alguma estrutura parasitária como larvas e ovos de helmintos, assim como cistos e oocistos de protozoários. Insetos, ácaros e larvas de vida livre que são apontados como importantes transportadores de agentes patogênicos também foram encontrados (OLIVEIRA DCS et al., 2012), o que reforça a necessidade de uma higienização adequada para deixar as alfaces em condições de consumo.

Ambos os casos apresentam dados preocupantes e sugestivos para melhorar a cadeia produtiva, desde a coleta até a comercialização. De acordo com a Agência de Vigilância Sanitária (ANVISA), as hortaliças têm que ser comercializadas livres de sujidades, larvas e parasitos (QUADROS RM et al., 2008).

Estudos similares, realizados em alfaces de hortas comunitárias demonstram os seguintes resultados, Mesquita DR et al. (2015), examinaram 120 amostras de alface-crespa (Lactuca sativa) oriundas de três hortas comunitárias de Teresina (PI) e evidenciaram uma positividade para estruturas parasitárias em 34,1\% (41/120) das amostras. Em Cuiabá (MT), Alves AS et al., (2013) mostraram que 66,7\% (30/45) das amostras de alfaces comercializadas nos supermercados foram positivas para a presença protozoários e helmintos, corroborando os resultados encontrados no nosso estudo.

O complexo de doenças causadas por parasitas intestinais expressa ainda números superlativos, haja vista que, a maioria destas enfermidades podem ser evitadas com medidas simples de higiene individual e coletiva. Ações de infraestrutura e educacionais promovidas pelos governos também são fontes importantes no combate ao problema que acomete milhares de pessoas. Entretanto, mesmo com impactos significativos nas condições de saúde, sociais e econômicas ainda são escassos os trabalhos com intenção de avaliar o padrão qualitativo que envolve as hortas distribuidoras de hortaliças para o meio comercial. Os parasitas constatados nesse trabalho são de importância médica, pois, apresenta espécies de caráter zoonótico, o que sugere contaminação fecal de origem humana e/ou animal nas alfaces estudadas (SANTANA LRR et al., 2006), como o demostrado na Tabela 3.

Tabela 3- Relação entre espécies e a positividade em supermercados e hortas comunitárias em Teresina PI.

\begin{tabular}{|c|c|c|c|c|c|c|c|c|c|c|c|c|c|c|}
\hline \multirow{3}{*}{ Espécies } & \multicolumn{8}{|c|}{ Supermercados } & \multicolumn{6}{|c|}{ Hortas comunitárias } \\
\hline & \multicolumn{2}{|c|}{ S1 (5) } & \multicolumn{2}{|c|}{ S2 (4) } & \multicolumn{2}{|c|}{ S3 (4) } & \multicolumn{2}{|c|}{ S4 (4) } & \multicolumn{2}{|c|}{$\mathrm{H} 1$ (5) } & \multicolumn{2}{|c|}{$\mathrm{H} 2(5)$} & \multicolumn{2}{|c|}{ H3 (5) } \\
\hline & $\mathrm{N}$ & $\%$ & $\mathrm{~N}$ & $\%$ & $\mathrm{~N}$ & $\%$ & $\mathrm{~N}$ & $\%$ & $\mathrm{~N}$ & $\%$ & $\mathrm{~N}$ & $\%$ & $\mathrm{~N}$ & $\%$ \\
\hline Ascaris sp. & 2 & 40 & - & . & 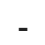 & . & 1 & 25 & 1 & 20 & 3 & 60 & - & 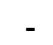 \\
\hline Ancylostoma sp. & 1 & 20 & 2 & 50 & 2 & 50 & 3 & 75 & - & 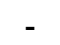 & 1 & 20 & 1 & 20 \\
\hline Strongyloides $s p$. & 1 & 20 & - & - & 1 & 25 & - & - & - & - & - & - & - & \\
\hline Entamoeba histolytica/díspar & 2 & 40 & 1 & 25 & 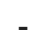 & - & 1 & 25 & 1 & 20 & 2 & 40 & 3 & 60 \\
\hline Giardia lamblia & 3 & 60 & 2 & 50 & 2 & 50 & 3 & 75 & - & - & 3 & 60 & 2 & 40 \\
\hline Isospora belli & & & 1 & 25 & & & & & - & - & & & 1 & 20 \\
\hline Eimeria sp. & 2 & 40 & 1 & 25 & 2 & 50 & 2 & 50 & 2 & 40 & 4 & 80 & 3 & 60 \\
\hline Entamoeba coli & 2 & 40 & 1 & 25 & 1 & 25 & 1 & 25 & 1 & 20 & 2 & 40 & 1 & 20 \\
\hline Endolimax nana & 4 & 80 & 2 & 50 & 3 & 75 & 2 & 50 & 3 & 60 & 4 & 80 & 2 & 40 \\
\hline lodamoeba butschlii & 1 & 20 & - & - & & - & 2 & 50 & - & - & 2 & 40 & - & - \\
\hline
\end{tabular}

Legenda: $\mathrm{SX}(\mathrm{n})$ = Supermercado $X$, com " $n$ " amostras; $\mathrm{HX}(\mathrm{n})=$ Horta comunitária $X$, com "n" amostras; $N=$ Quantidade de amostras positivas; $\%=$ percentual de positividade.

Fonte: Silva J, Moura VG, Silva MJM, et al., 2016.

Aponta-se para a positividade das amostras, a vulnerabilidade pela contaminação de origem fecal no solo da horta, contaminando as hortaliças. As práticas agrícolas, condições ecológicas, transporte, manuseio, condições de armazenamento e comercialização são fatores que também contribuem para a ocorrência de agentes contaminantes para esta hortaliça (QUADROS RM, et al., 2008).

Nas alfaces das hortas comunitárias a ocorrência de enteroparasitas foi maior. Segundo Montanher CC (2007) é comum encontrar números mais expressivos de biocontaminantes entre verdureiros (horticultores) do que em supermercados, pois nestes ao serem comercializadas, passam por algum tipo de seleção e lavagem, eliminando as sujidades e consequentemente, os parasitos. A Tabela 2 expõe a biocenose parasitária encontrada nas amostras. 
Nascimento MP, et al., (2016) ao realizarem análises de 22 amostras de alface-crespa coletadas em feira livre da cidade de Barro (CE), evidenciaram que destas, 90,91\% (20/22) estavam contaminadas com larvas e ovos de helmintos. Ovos de Ascaris lumbricoides estavam presentes na maioria das amostras (72,7\%), seguido por ovos de Schistosoma mansoni (50\%)e larvas de Ancylostoma sp. (27,3\%).

Em estudo realizado em Teresina (PI), das 120 amostras de alface-crespa coletadas em hortas comunitárias, $41(34,1 \%)$ apresentaram estruturas parasitárias, com destaque de ocorrência para cistos de Balantidium sp., tendo na sequência larvas dos gêneros Strongyloides sp. e Ancylostoma sp (MESQUITA DR, et al., 2015).

Em relação aos helmintos encontrados nas análises. Estes vermes apresentam caracteres adaptativos que os favorecem a tal exposição. São animais que geralmente circulam a partir de veiculação hídrica e/ou alimentos contaminados e apresentam ciclo monóxeno, não necessitam abrigar-se em dois ou mais hospedeiros diferentes para completar o seu ciclo biológico, e por serem vermes adaptados ao endoparasitismo, possuem uma estrutura envoltória composta por colágeno (película) que os protege contra líquidos enzimáticos de seu hospedeiro (BARUFFALD R, et al.,1984).

Sua habilidade em forragear vários tipos de fontes alimentícias, aliada às suas estruturas adaptativas, permite sua flexibilidade de ocorrência em vários produtos de origem animal e vegetal, entre estes, a alface. Excetuando-se o filo Arthropoda, os animais do filo Nematoda constituem o grupo mais diversificado na escala zoológica (BRUSCA RC e BRUSCA GJ, 2007).

Entre os protozoários foram encontrados organismos comensais (não patogênicos) e parasitas (patogênicos). Entre os comensais têm-se as Entamoeba coli, Endolimax nana e lodamoeba bustchlii, embora não patogênicas, são bioindicadoras sinalizando para a contaminação fecal da água utilizada no plantio, más condições de higienização ou baixa qualidade higiênico-sanitária durante o transporte, armazenamento e comercialização dos pés de alface (MESQUITA VCL, et al.,1999).

Os protozoários patogênicos estão representados pelo complexo Entamoeba histolytica/dispar, que conduz o infectado a um quadro clínico que pode ter como consequência um leve desconforto abdominal e nos casos invasivos levar um ser humano a óbito. Giardia sp. que é o principal responsável por desnutrição em crianças em todo o mundo e Isospora belli, um coccídeo emergente que é assintomático na maioria dos casos. No entanto, nos demais casos, há diarreia, cólicas abdominais e febre, quando a isosporíase ocorre em indivíduos imunocomprometidos, e neste caso, as manifestações tornam-se crônicas (VIANA SGF e SOGAYAR MITL, 2011; FRANCO RMB, 2011).

\section{CONCLUSÃO}

As alfaces comercializadas em Teresina-PI por horticultores e em supermercados apresentaram altos índices de contaminação por enteroparasitos (66,5\%), esse percentual é extremamente elevado considerando que as amostras para consumo têm que se apresentar totalmente livres de sujidades. As amostras obtidas a partir dos horticultores apresentaram-se com maior índice de contaminação, porém a positividade das amostras para ambos os setores indica que estratégias de higienização têm que ser melhor implementadas visando o consumo saudável desta hortaliça para a população da área de estudo e práticas de maior vigilância por parte dos órgãos de fiscalização.

\section{REFERÊNCIAS}

1. ADANI AAV, DUTRA MBL. Análise da eficácia do vinagre como sanitizante na alface. Revista Eletrônica Acervo Saúde, 2011; 3: 134-144.

2. ALVES AS, et al. Parasitos em alface-crespa (Lactuca sativa L.), de plantio convencional, comercializada em supermercados de Cuiabá, Mato Grosso, Brasil. Revista de Patologia Tropical, 2013; 42(2): 217-229.

3. BARUFFALDI R, et al. Tratamento químico de hortaliças poluídas. Revista Saúde Pública, 1984; $18(3)$ : $224-234$.

4. BRUSCA RC, BRUSCA GJ. Invertebrados. Segunda edição. Editora Guanabara- Koogan, Rio de Janeiro. 2007. 968p.

5. COELHO WMD, et al. Ocorrência de parasitos gastrintestinais em amostras fecais de felinos no município de Andradina. Revista Brasileira de Parasitologia Veterinária, 2009; 18(2): 46-49, 
6. COSTANTIN BS, et al. Avaliação da contaminação parasitológica em alfaces: um estudo no sul do Brasil. Revista Fasem Ciências, 2013; 3 (1): 9-22.

7. FALAVIGNA LM, et al. Qualidade de hortaliças comercializadas no noroeste do Paraná, Brasil. Parasitologia Latinoamericana, 2005.60(3-4): 144-149.

8. FERRO JJB, et al. Avaliação parasitológica de alfaces (Lactuca sativa) comercializadas no município de Tangará da Serra, Mato Grosso, Brasil. Revista de Patologia Tropical, 2012; 41(1): 47-54.

9. FRANCO RMB. Sarcocytis, Cystoisospora e Cryptosporidium. In: Neves D. P, Melo A. L, Linardi P. M, Vitor R. W, editores. Parasitologia Humana. 12ª ed. São Paulo: Atheneu; 2011. p. 189-198.

10. FREITAS AA, et al. Avaliação parasitológica de alfaces (Lactuca sativa) comercializadas em feiras livres e supermercados do município de Campo Mourão, Estado do Paraná. Acta Scientiarum. Biological Sciences. 2004;26(4):381-384.

11. GONDIM A (Ed.). Alface. Catálogo Brasileiro de Hortaliças, Brasília,2010; 60p.

12. GREGÓRIO DS, et al. Estudo da contaminação por parasitas em hortaliças da região leste de São Paulo. Science in Health, 2012; 3(2): 96-103.

13. MELO ACFL, et al. Contaminação parasitária de alfaces e sua relação com enteroparasitoses em manipuladores de alimentos. Revista Trópica - Ciências Agrárias e Biológicas, 2011; 5(3): 47-52.

14. MESQUITA DR, et al. Ocorrência de parasitos em alface-crespa (Lactuca sativa L.) em hortas comunitárias de Teresina, Piauí, Brasil. Revista de Patologia Tropical, 2011; 44(1): 67-76.

15. MESQUITA VCL, et al. Contaminação por enteroparasitas em hortaliças comercializadas nas cidades de Niterói e Rio de Janeiro, Brasil. Revista da Sociedade Brasileira de Medicina Tropical. 1999;32(4): 363-366.

16. MONTANHER CC, et al. Avaliação parasitológica em alfaces (Lactuca sativa) comercializadas em restaurantes self service por quilo, da cidade de Curitiba, Paraná, Brasil. Estudos de Biologia, Curitiba, 2007; 29(66): 63-71.

17. NASCIMENTO MP, et al. Avaliação parasitológica da alface (Lactuca sativa L.) comercializada na feira livre de Barro - CE, Brasil. Caderno de Cultura e Ciências, 2016; 15(2): 1-11.

18. NEVES DP. Parasitologia Humana. 12 ${ }^{\underline{a}}$ ed. São Paulo: Atheneu, 2011. 546p

19. OLIVEIRA DCS, et al. Avaliação parasitológica em amostras de alfaces (Lactuca sativa) Comercializadas em supermercados de Ipatinga, Minas Gerais. Nutrir gerais2012; 6(11): 933-944.

20. QUADROS RM, et al. Parasitos em alfaces (Lactuca sativa) de mercados e feiras livres de Lages - Santa Catarina. Revista Ciências da Saúde, 2008; 1(2): 78-84.

21. ROCHA MO, MELO RT. Exame parasitológico de fezes. In: Neves DP, Melo AL, Linardi PM, Vitor RW, editores. Parasitologia Humana. $12^{\underline{a}}$ ed. São Paulo: Atheneu; 2011. p. 509-527.

22. SANTANA LRR, et al. Qualidade física, microbiológica e parasitológica de alfaces (Lactuca sativa) de diferentes sistemas de cultivo. Ciência e Tecnologia de Alimentos. 2006;26(2):264-269.

23. SANTARÉM VA, et al. contaminação de hortaliças por endoparasitas e salmonella spp. em Presidente Prudente, São Paulo, Brasil. Colloquium Agrariae, 2012; 8(1): 18-25.

24. SANTOS SA, MERLINI LS. Prevalence of enteroparasitosis in the population of Maria Helena, Paraná State. Ciências \& Saúde Coletiva, 2010;15(3):899-905.

25. SOARES B. CANTOS GA. Qualidade parasitológica e condições higiênico sanitárias de hortaliças comercializadas na cidade de Florianópolis, Santa Catarina, Brasil. Revista Brasileira de Epidemiologia. 2005; 8(4):377-384.

26. TAKAYANAGUI OM, et al. Análise da cadeia de produção de verduras em Ribeirão Preto, SP. Revista da Sociedade Brasileira de Medicina Tropical, 2006; 39(2): 224-226.

27. VIANA SGF, SOGAYAR MITL. Giárdia. In: Neves DP, Melo AL, Linardi PM, Vitor RW, editores. Parasitologia Humana. 12 ${ }^{\mathrm{a}}$ ed. São Paulo: Atheneu; 2011. p.129-136.

28. VIEIRA JN, et al. Parasitos em hortaliças comercializadas no sul do Rio Grande do Sul, Brasil. Revista Ciência Médica e Biológica, Salvador, v.12, n.1, p.45-49, jan. /abr. 2013. 